\title{
Colloidal quantum dot absorption enhancement in flexible Fano filters
}

\author{
Li Chen, ${ }^{1}$ Hongjun Yang, ${ }^{1}$ Zexuan Qiang, ${ }^{1}$ Huiqing Pang, ${ }^{2}$ Lei Sun, ${ }^{2}$ Zhengiang $\mathrm{Ma},{ }^{2}$ \\ Ryan Pate, ${ }^{3}$ Adrienne Stiff-Roberts, ${ }^{3}$ Shuai Gao, ${ }^{4}$ Jian Xu, ${ }^{4}$ Gail J. Brown, ${ }^{5}$ and \\ Weidong Zhou ${ }^{1, \text { a) }}$ \\ ${ }^{1}$ Department of Electrical Engineering, NanoFAB Center, University of Texas at Arlington, Texas 76019, USA \\ ${ }^{2}$ Department of Electrical and Computer Engineering, University of Wisconsin-Madison, \\ Wisconsin 53706, USA \\ ${ }^{3}$ Department of Electrical and Computer Engineering, Duke University, Durham, \\ North Carolina 27708, USA \\ ${ }^{4}$ Department of Engineering Science and Mechanics, Pennsylvania State University, University Park, \\ Pennsylvania 16802, USA \\ ${ }^{5}$ Air Force Research Laboratory, Materials and Manufacturing Directorate, Wright Patterson AFB, \\ Ohio 45433-7707, USA
}

(Received 29 December 2009; accepted 7 February 2010; published online 25 February 2010)

\begin{abstract}
We report here modified absorption property of colloidal quantum dots (CQDs) inside flexible Fano filters - made of patterned single crystalline silicon nanomembrane transferred onto flexible plastic substrates. Enhanced optical absorption was obtained both experimentally and theoretically, when the CQD absorption peak spectrally overlaps with Fano resonance peak. On the other hand, suppressed absorption was observed when the Fano resonance has no spectral overlap with the CQD absorption bands. (C) 2010 American Institute of Physics. [doi:10.1063/1.3337095]
\end{abstract}

Owing to the ability to manipulate photon density of states (DOS) and to alter light-matter interaction, photonic crystals (PC) offer a disruptive platform for nanophotonics. ${ }^{1}$ In addition to strong spontaneous emission control, ${ }^{2,3}$ the PC structures can also lead to strong optical absorption modulation. Modified absorption characteristics in two-dimensional dielectric photonic crystal slab (PCS) cavities have been reported by various groups previously. ${ }^{4-8}$ Most of these studies were based on epitaxial III-V quantum dot system.

Recently, great attention has also been paid to Fano resonances, a class of "leaky" modes in PCS. ${ }^{9-14}$ These resonances are standing electromagnetic waves that are guided with the electric field distribution confined within PCS but are also strongly coupled to out-of-the-plane radiation modes due to phase matching provided by the periodic lattice structure of the PCS. We have reported surface-normal optical Fano resonance filters, based on single crystalline semiconductor nanomembranes (NMs) that are transferred to both rigid glass substrates and to flexible polyethylene terephthalate (PET) substrates. ${ }^{12,13}$ The unique angular-, spectral-, and polarization-dependent properties of these Fano filters have also been investigated extensively, indicating that Fano resonance-based PCS is a promising platform for various nanophotonic devices. ${ }^{14}$

By incorporating dispersive medium inside Fano resonance cavities, it is feasible to realize ultra-compact infrared photodetectors with spectrally-selective absorption enhancement properties on any substrates for any wavelengths. We have earlier reported theoretical work on enhanced IR absorption in defect-free PC cavities, via Fano resonances, due to spectrally-selective light-matter interaction in the cavities. ${ }^{15,16}$ We report here direct absorption measurements on solution processed colloidal quantum dots (CQDs) in patterned Si NM PC Fano resonance cavities that are transferred to flexible plastic PET substrates.

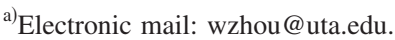

The Fano resonance filter structure was designed and fabricated on a silicon-on-insulator (SOI) substrate using standard e-beam lithography and dry etching processes. ${ }^{13}$ Shown in Figs. 1(a) and 1(b) are the cross-sectional view and the top view scanning electron micrographs (SEM), respectively, for the fabricated PC structures on the SOI. The thickness of the top Si NM PC layer $(t)$ is $250 \mathrm{~nm}$. The squarelattice air-hole PC structure has a period $(a)$ of $600 \mathrm{~nm}$, and the air hole radius $(r)$ of $114 \mathrm{~nm}$. The structure was subsequently transferred to a flexible PET substrate, using a wet transfer process. A micrograph of the finished device is shown in Fig. 1(c). Finally, colloidal $\mathrm{PbSe}$ or $\mathrm{PbS}$ quantum dots (CQDs) were back-filled inside the air holes of the transferred SiNM PC region. Shown in Fig. 1(d) is a typical transmission electron micrograph (TEM) image of nanocrystal PbSe CQDs used in the study here. PbSe CQDs were
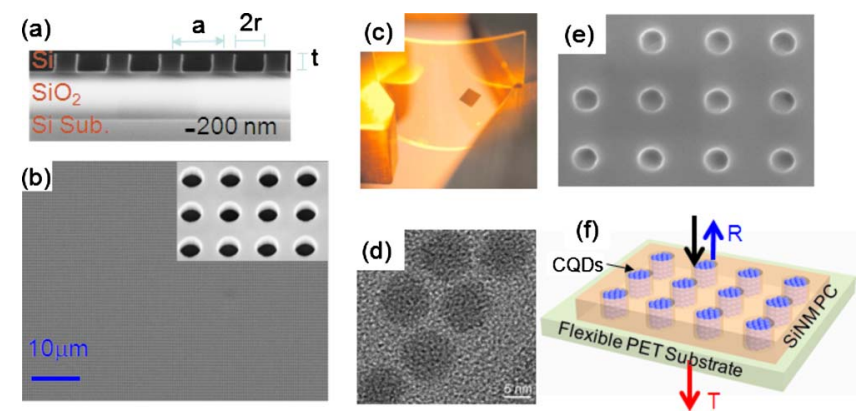

FIG. 1. (Color online) (a) Cross-sectional view and (b) Top view scanning electron micrographs (SEMs) of fabricated photonic crystal Fano filter structure based on silicon-on-insulator (SOI) wafers, where $r, a$, and $t$ are air hole radius, lattice constant, and Si layer thickness, respectively; (c) Micrograph of flexible Fano filters based on transferred single crystalline Si nanomembrane (SiNM) on flexible PET substrates; (d) Transmission electron micrograph (TEM) of nanocrystal colloidal PbSe quantum dots (CQDs); (e) Top view SEM image of Fano filter filled with PbSe CQDs; and (f) Schematic of SiNM based Fano filters filled with CQDs on flexible substrates. Surface normal optical measurement is also shown for both transmission $(T)$ and reflection $(R)$. 


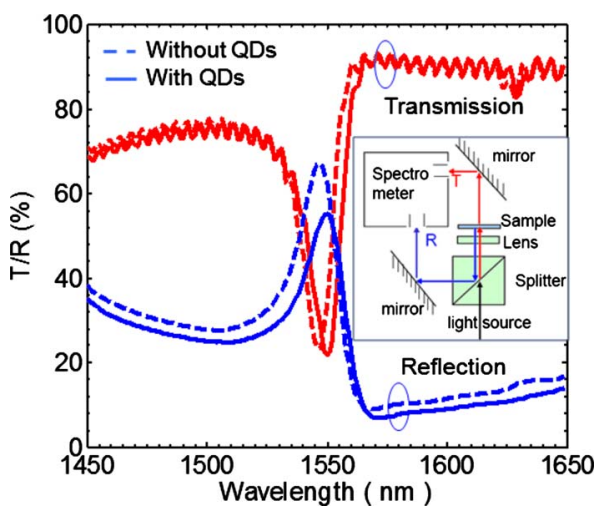

FIG. 2. (Color online) Measured transmission and reflection spectra for Fano filter without and with PbSe CQDs, with the inset shown schematically the experimental setup for the simultaneous transmission/reflection measurement of the Fano filter.

synthesized with a noncoordinating solvent technique, ${ }^{17}$ with the target wavelengths covering different Fano resonance regions, ranging from 1450 to $1600 \mathrm{~nm}$.

Shown in Fig. 1(e) is the top view of a SEM image of a Si NM PC Fano filter filled with PbSe CQDs. A schematic of the final structure under investigation is shown in Fig. 1(e), for which transmission/reflection measurements are carried out along the surface-normal direction.

The experimental setup is shown in the inset of Fig. 2. A focused broadband light source is generated from a quartz tungsten halogen (QTH) lamp. The beam transmitted through the Fano filter sample was collected by one entrance slit of a spectrometer, and the beam reflected from the sample was sent to the other entrance slit via a beam splitter. The measured transmission spectrum $(T)$ was obtained by normalizing the measured transmission spectral intensity data for the Fano filters to the that for the background (air). The measured reflection spectrum $(R)$ was obtained by normalizing the measured reflection spectral intensity data for the Fano filters to that for the reference mirror (gold coated), which has a reflectivity close to $100 \%$. Care was taken to ensure the simultaneous measurements of the absolute transmission and the reflection spectra for the sample, under exactly the same conditions. In this case, the absolute absorption $(A)$ can be derived based on the equation $A=1-T-R$.

Shown in Fig. 2 are a set of measured transmission/ reflection spectra for the Fano filters without and with CQDs. At the spectral regime of interest (around $1550 \mathrm{~nm}$ ), the absorption due to $\mathrm{Si}$ is negligible, i.e., $A=0$. So all measured absorption is largely associated with the absorption of the incorporated CQD. A distinctive feature shown in Fig. 2 is the spectral redshift when incorporating CQDs, with the peak resonance shifted from 1547 to $1550 \mathrm{~nm}$. This is due to the increase of the effective index in the air hole region of the patterned Fano filters. Based on the simulations using the rigorous coupled-wave analysis (RCWA) method, the effective index within air holes $\left(\mathrm{n}_{\mathrm{h}}\right)$ of the patterned SiNMs was determined to be 1.09 , corresponding to a $4 \%$ fill factor of CQDs.

After CQDs deposition, the measured transmission intensity of the resonance mode was reduced by $6.2 \%$ and the quality factor $(\mathrm{Q})$ of the Fano resonance was degraded by $8.6 \%$. This also agrees well with the RCWA simulated Q-factor degradation of $5.1 \%$. On the other hand, the mea-

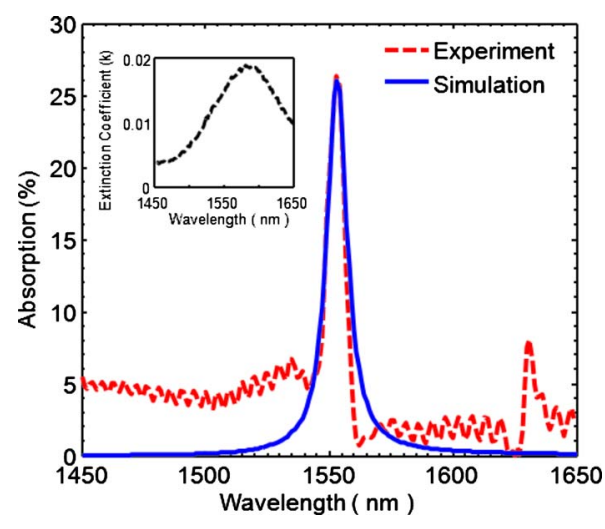

FIG. 3. (Color online) Experimental and simulated absorption characteristics for the Fano filter filled with CQDs. Shown in the inset is the spectra of CQD extinction coefficient, $\mathrm{k}$.

sured reflected intensity at Fano resonance was significantly reduced by $18 \%$. The Q-factor was significantly degraded by $17 \%$. Both of these distinctive features in the measured transmission/reflection spectra agree well with the simulation results.

The absorption data for the Fano filter was directly extracted from the transmission and the reflection based on the equation $A=1-T-R$, where $A, T$, and $R$ are absorption, transmission, and reflection, respectively. The measured absorption spectrum was shown as the dash line in Fig. 3, where we found a sharp peak at the shifted Fano resonance regime $(1550 \mathrm{~nm})$. The simulated absorption spectrum was also shown in Fig. 3 (solid line), with the fitting extinction coefficient $(k)$ shown in the inset of Fig. 3. The measured and the simulated spectra match very well with each other.

To further illustrate the interactions between the Fano filters and the CQDs, and also to quantify the changes in the absorption due to the interactions, we introduce an absorption enhancement factor parameter. It is defined as the ratio of the absorption of the CQDs inside Fano filters to that of the CQDs without Fano filters. Figures 4(a) and 4(b) show the absorption spectra of three wavelength QDs and the transmission spectra of three Fano filters, respectively. Using these QDS and filters, three different cases were studied. For Case 1 (QD1 and Filter 1): (with the data also shown in Fig. 2 and Fig. 3), there is a very good spectral overlap between the Filter 1 resonance peak and the QD1 absorption around $1550 \mathrm{~nm}$. This overlap leads to an absorption enhancement factor of 9 at the resonance location of Filter 1, as shown in Fig. 4(c). In the second case (Filter 2 and QD2), a Fano filter sample was fabricated with Fano resonance at $1468 \mathrm{~nm}$, by increasing the air hole radius (r) to $149 \mathrm{~nm}$. This sample was filled with another spectrally-matched PbSe CQDs (QD2) with their absorption peak at around $1450 \mathrm{~nm}$. Following the same procedure, the absorption enhancement factor was derived and the data were plotted in Fig. 4(c). An absorption enhancement factor of 18 for this sample was obtained, which may be due to the combined effects of a larger air fill factor, a higher quality factor of Fano resonance as well as a higher quantum efficiency of CQDs in this case.

Finally, another set of Fano filters (Filter 3) was fabricated with Fano resonance of $1564 \mathrm{~nm}$, by reducing the air hole size to $103 \mathrm{~nm}$ in radius. CQDs with absorption peak at $1640 \mathrm{~nm}$ (QD3) were used here, which have no absorption at $1564 \mathrm{~nm}$. In this case, there is no absorption observed around 

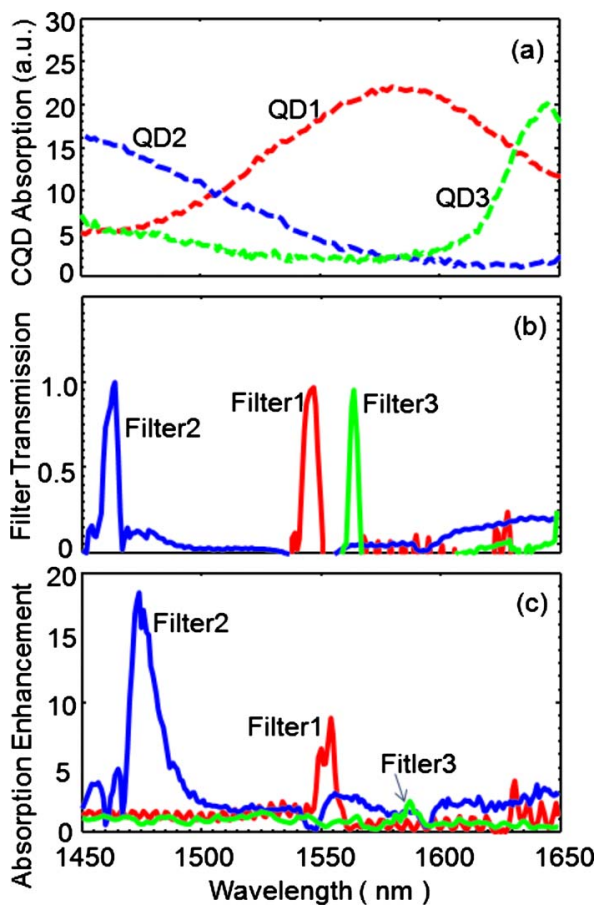

FIG. 4. (Color online) (a) Three sets of CQDs with different absorption peak locations; (b) three different Fano filters with different resonance locations; and (c) absorption enhancement factors for these three sets of samples.

$1564 \mathrm{~nm}$ spectral regime. By comparing these three cases, it clearly indicates that the enhanced absorptions at different Fano resonance locations are originated from the strong interactions between the absorptive medium (CQDs in this case) and the spectrally matched Fano resonances.

It is also worth noting that the quality factors of Fano resonance in our experiments were far from optimal. According to our earlier theoretical work, one or two orders of absorption enhancements can be expected for the Fano filters with optimized quality factors. ${ }^{15}$ In addition, the fill factor of CQDs into the air holes of PC cavities can also be further increased.

Another interesting feature for the photodetectors based on Fano resonance filters is the ability of dispersion engineering for the desired angular and polarization properties. ${ }^{13,14}$ We observed experimentally a blueshift (4.5 $\mathrm{nm} /{ }^{\circ}$ ) in absorption resonances for transverse electrical (TE) mode, with the increase of incident angles up to $20^{\circ}$ off surface-normal incidence. At the same time, the absorption resonance for transverse-magnetic (TM) mode does not shift much. These experimental results agree well with the simulated absorption properties, based on the RCWA technique. The results also agree well with previously reported results on Fano resonance filters. ${ }^{14}$ The spectra shift is largely dependent on the polarization states of incident beam, the direction of the incident beam and, the phase matching condi- tions. It is feasible to have different spectral shifting characteristics, either redshifts or blueshifts, at various shift rates ranging 0.5 to $5 \mathrm{~nm} /{ }^{\circ} .^{14}$

In conclusion, $\mathrm{PbSe} / \mathrm{PbS} \mathrm{CQD}$ absorption characteristics were directly measured inside the air holes of Si Fano resonance filters on flexible PET substrates. The experimental results agree well with the simulation results. Significant absorption enhancements can be found at Fano resonance when the CQD absorption spectrally overlaps with the Fano filter resonance. The study will lead to a new class of photodetectors with desired angular-, spectral-, and polarizationdependent properties and such devices can be widely used in optical communications, hyper-spectral imaging systems and flexible photonics.

This work was supported by U.S. AFOSR Nano Programs (Grant No. FA9550-06-1-0482), AFOSR MURI program (Grant No. FA9550-08-1-0337), AFRL CONTACT program (Grant No. FA 8650-07-2-5061), and by National Science Foundation under Grant No. DMI-0625728. The authors also acknowledge the fabrication support from the University of Texas at Arlington NanoFAB Center and the University of Texas Austin MRC center, part of the NSF NNIN sites.

${ }^{1}$ S. Noda, T. Baba, and O. Industry, Roadmap on Photonic Crystals (Springer, New York, 2003).

${ }^{2}$ E. Yablonovitch, Phys. Rev. Lett. 58, 2059 (1987).

${ }^{3}$ S. Noda, M. Fujita, and T. Asano, Nat. Photonics 1, 449 (2007).

${ }^{4}$ K. T. Posani, V. Tripathi, S. Annamalai, N. R. Weisse-Bernstein, S. Krishna, R. Perahia, O. Crisafulli, and O. J. Painter, Appl. Phys. Lett. 88, 151104 (2006).

${ }^{5}$ S. Schartner, S. Golka, C. Pflugl, W. Schrenk, A. M. Andrews, T. Roch, and G. Strasser, Appl. Phys. Lett. 89, 151107 (2006).

${ }^{6}$ W. Zhou, L. Chen, Z. Qiang, and G. J. Brown, J. Nanophotonics 1, 013515 (2007).

${ }^{7}$ H. Yang, L. Chen, Z. Qiang, W. Zhou, W. Zhang, A. Stiff-Roberts, S. Krishna, and G. J. Brown, 20th Annual Meeting of the IEEE Lasers and Electro-Optics Society (IEEE, New York, 2007), pp. 36-37.

${ }^{8}$ J. K. Yang, M. K. Seo, I. K. Hwang, S. B. Kim, and Y. H. Lee, Appl. Phys. Lett. 93, 211103 (2008).

${ }^{9}$ S. Fan and J. D. Joannopoulos, Phys. Rev. B 65, 235112 (2002).

${ }^{10}$ A. Rosenberg, M. Carter, J. Casey, M. Kim, R. Holm, R. Henry, C. Eddy, V. Shamamian, K. Bussmann, S. Shi, and D. W. Prather, Opt. Express 13, 6564 (2005)

${ }^{11}$ Y. Ding and R. Magnusson, Opt. Express 12, 5661 (2004).

${ }^{12}$ H. Yang, Z. Qiang, H. Pang, Z. Ma, and W. D. Zhou, Electron. Lett. 44 858 (2008).

${ }^{13}$ Z. Qiang, H. Yang, L. Chen, H. Pang, Z. Ma, and W. D. Zhou, Appl. Phys. Lett. 93, 061106 (2008).

${ }^{14}$ L. Chen, Z. Qiang, H. Yang, H. Pang, Z. Ma, and W. D. Zhou, Opt. Express 17, 8396 (2009).

${ }^{15}$ Z. Qiang, W. D. Zhou, M. Lu, and G. J. Brown, Proc. SPIE 6901, 69010F (2008).

${ }^{16}$ W. D. Zhou, H. Yang, Z. Qiang, L. Chen, and G. J. Brown, Proc. SPIE 7095, 709507 (2008).

${ }^{17}$ D. Cui, J. Xu, T. Zhu, G. Paradee, S. Ashok, and M. Gerhold, Appl. Phys. Lett. 88, 183111 (2006). 